\title{
Diagnostic performance of AccuFFRangio in the functional assessment of coronary stenosis compared with pressure wire-derived fractional flow reserve
}

\author{
Jun Jiang ${ }^{1 \#}$, Lijiang Tang ${ }^{2 \#}$, Changqing $\mathrm{Du}^{2}$, Xiaochang Leng ${ }^{3}$, Jingsong $\mathrm{He}^{3}$, Yumeng $\mathrm{Hu}^{3} \wedge$, Liang Dong ${ }^{1}$, \\ Yong Sun ${ }^{1}$, Changling Li ${ }^{1}$, Jianping Xiang ${ }^{3}$, Jian'an Wang ${ }^{1}$ \\ ${ }^{1}$ Department of Cardiology, The Second Affiliated Hospital, Zhejiang University School of Medicine, Hangzhou, China; ${ }^{2}$ Department of Cardiology, \\ Zhejiang Hospital, Hangzhou, China; ${ }^{3}$ ArteryFlow Technology Co., Ltd., Hangzhou, China
}

Contributions: (I) Conception and design: All authors; (II) Administrative support: J Xiang, J Wang, C Li, X Leng; (III) Provision of study materials or patients: All authors; (IV) Collection and assembly of data: All authors; (V) Data analysis and interpretation: All authors; (VI) Manuscript writing: All authors; (VII) Final approval of manuscript: All authors.

"These authors contributed equally to this work.

Correspondence to: Jianping Xiang, PhD. ArteryFlow Technology Co., Ltd., 459 Qianmo Road, Hangzhou 310051, China. Email: jianping.xiang@arteryflow.com; Jian'an Wang, MD. Department of Cardiology, The Second Affiliated Hospital, Zhejiang University School of Medicine, 88 Jiefang Road, Hangzhou 310009, China. Email: wja@zju.edu.cn.

Background: Non-invasive fractional flow reserve (FFR) has been increasingly used in the clinical workflow to assist clinical decision-making for percutaneous coronary intervention (PCI). This clinical study evaluates the diagnostic accuracy of coronary stenosis assessed by a non-invasive FFR analysis method (termed AccuFFRangio) based on invasive coronary angiography (ICA). It is a blinded, self-controlled, retrospective, and dual-center clinical investigation study.

Methods: Coronary angiography data and the related information of 320 patients with 320 vessels were collected, and AccuFFRangio was used to assess the FFR for these patients. Compared with the wiremeasured FFR values, we evaluated AccuFFRangio performance by its accuracy, sensitivity, specificity, positive predictive value (PPV), and negative predictive value (NPV).

Results: The diagnostic accuracy, sensitivity, specificity, PPV, and NPV for AccuFFRangio in identifying hemodynamically significant coronary stenosis were 93.3\%, 92.6\%, 93.5\%, 84.3\%, and 97.1\%, respectively. The direct correlation between computed AccuFFRangio and measured FFR was $0.812(\mathrm{P}<0.001)$, and the area under the receiver operating characteristic curve (AUC) value of AccuFFRangio was 0.96.

Conclusions: This clinical study demonstrates the efficiency and accuracy of AccuFFRangio for clinical implementation when using invasive wire-measured FFR as a reference. Further validation is required in a large prospective multicenter study.

Keywords: AccuFFRangio; fractional flow reserve (FFR); invasive coronary angiography (ICA); stenosis

Submitted Apr 30, 2021. Accepted for publication Aug 26, 2021.

doi: 10.21037/qims-21-463

View this article at: https://dx.doi.org/10.21037/qims-21-463

\footnotetext{
$\wedge$ ORCID: 0000-0002-0779-9172.
} 


\section{Introduction}

Coronary computed tomography angiography (CTA) is a routine examination for patients with suspected coronary artery disease (CAD), and it has been the main factor influencing decision-making and patient management for a long time. However, it has been argued that coronary CTA could increase the rate of downstream examination, including invasive coronary angiography (ICA) and further intervention (1). This is mainly because of the inaccuracy of the angiographic assessment of coronary stenosis. Furthermore, it was reported that over $50 \%$ of severe stenoses determined by CTA were overestimated, which means numerous unnecessary invasive angiography referrals (2). Invasive fractional flow reserve (FFR) can be used to assess patients with functional myocardial ischemia accurately (3). It has demonstrated superior performance in guiding revascularization; thus, it has a class Ia recommendation in society guidelines (4-6). However, invasive FFR examination requires the use of a pressure wire to pass through the stenosis lesion and measure the pressure at the distal end of the stenosis, typically $2-3 \mathrm{~cm}$ away. At the same time, adenosine or ATP injection is required to induce hyperemia in the distal microvascular system of the patient. Manipulation of the pressure wire could be risky due to the possibility of vessel wall injury or plaque activation during surgery. Additionally, it could be dangerous when treating patients with adenosine intolerance.

Moreover, the relatively high price of the wire-measured FFR limits invasive FFR examination in clinical practice. With the rapid development of computational FFR, some non-invasive (7-9) or non-pressure wire (10-12) FFR evaluation methods based on the combination of coronary angiography and computational fluid dynamics (CFD) have been developed (13). Non-invasive FFR based on coronary CTA imaging was first developed (7-9). Through a series of head-to-head clinical studies and real-world application studies $(14,15)$, it has been established as a more convenient and economical method for evaluating coronary artery function in addition to invasive FFR examination. Subsequently, FFR based on coronary angiography without pressure-wire has also developed rapidly. Through a series of clinical trials $(10,16-18)$, it has been proven that the functional assessment of patients with coronary stenosis can be more accurate by using 2 angiograms greater than $25^{\circ}$ apart through numerical calculation of pressure drop. This process completes the analysis in about 5 minutes, which is in line with the time required at the catheter room.
This study used coronary angiography to calculate the average volume flow during hyperemia using TIMI frame count combined with the three-dimensional (3D) target vessel. By applying CFD theory, a new FFR calculation method, AccuFFRangio, was proposed. The accuracy of the new wire-free FFR calculation method in evaluating functional ischemia of coronary stenosis was studied by using a retrospective cohort from 2 centers with invasive FFR as a reference.

We present the following article in accordance with the STARD reporting checklist (available at https://dx.doi. org/10.21037/qims-21-463).

\section{Methods}

\section{Patient population}

This study is a blinded, self-controlled, retrospective, and dual-center clinical investigation. The study was conducted in accordance with the Declaration of Helsinki (as revised in 2013). Patients with suspected and known CAD, who had undergone invasive FFR measurement between January 2018 and September 2019, were enrolled in this study. The study was approved by the Ethics Committees of The Second Affiliated Hospital, Zhejiang University School of Medicine and Zhejiang Hospital, and individual consent for this retrospective analysis was waived. The exclusion criteria were as follows: (I) lack of 2 optimal angiographic projections at least $25^{\circ}$ apart; (II) overlapping interrogated vessels with too much shortening without preferred references in proximal or distal vessels; (III) insufficient injected contrast for quantitative coronary angiography (QCA) analysis; (IV) location of the target lesion at the ostium of the left or right coronary artery; (V) acute myocardial infarction within 72 hours; (VI) severe asthma or severe chronic obstructive pulmonary disease; (VII) allergy to contrast media or adenosine; (VIII) atrial fibrillation.

Figure 1 presents the study enrollment flow chart. A total of 320 patients with 320 vessels (The Second Affiliated Hospital, Zhejiang University School of Medicine: 237 patients; Zhejiang Hospital: 83 patients) were included in this clinical study from January 2018 and September 2019. Due to the incomplete data from 10 patients, 310 patients went through the ICA procedure and pressure-wire FFR measurement. Among them, 12 patients were excluded due to predefined exclusion criteria, including too much vessel overlap, low image quality, 2 projections less than $25^{\circ}$, excessive pressure-wire drift, and technical issues. In the 


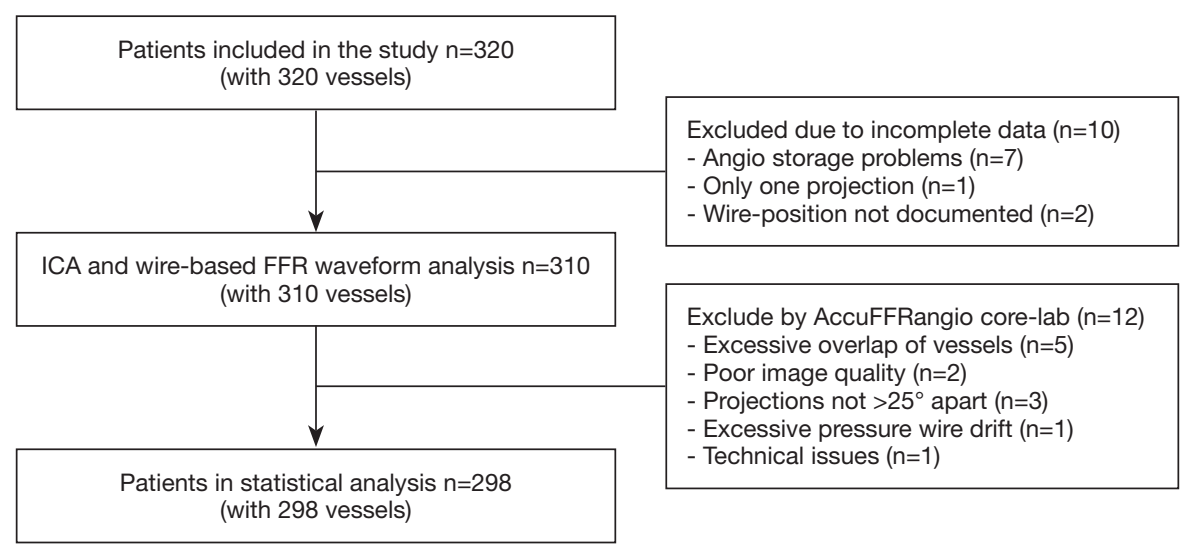

Figure 1 Patient flow chart of the study.

end, 298 patients with 298 vessels were included in the final analysis.

\section{ICA and two-dimensional (2D)-QCA analysis}

ICA was performed using the X-ray system (Allura Xper FD20/10; PHILIPS Medical Systems, the Netherlands). These angiographic images were recorded at a rate of 15 frames per second. The contrast medium was manually intensively and stably injected through a pump at a speed of approximately $4 \mathrm{~mL} / \mathrm{s}$. 2D-QCA was performed using Angiogram QCA software (Allura Xper FD20/10; PHILIPS Medical Systems, Netherlands).

\section{Wire-based FFR measurement}

The FFR at the distal end of the coronary stenosis in all patients was measured using a coronary pressure wire (St. Jude Medical, St. Paul, Minnesota, USA). After calibration and equalization, the pressure wire was advanced to the distal end of the stenosis. The concentration of intravenous adenosine triphosphate was $150-180 \mu \mathrm{g} / \mathrm{kg} / \mathrm{min}$, which was used to induce maximum hyperemia of the coronary microvascular system. At the same time, the distal coronary artery pressure at the pressure sensor and the proximal pressure at the coronary ostium was recorded. Then, the pressure sensor was pulled back to the proximal end of the catheter to check or correct the pressure drift.

\section{AccuFFRangio computation}

AccuFFRangio was computed with the AccuFFRangio V1.0 software (ArteryFlow Technology, Hangzhou, China) by participating physicians and technicians blinded to FFR. Two angiographic images with projections $>25^{\circ}$ apart at the end-diastolic frame were selected for $3 \mathrm{D}$ reconstruction of the segmented vessel. Then, the TIMI frame count was performed in an angiographic run to calculate the flow velocity under baseline conditions. AccuFFRangio distribution could be calculated through the pressure drop equation (19) (Figure 2) based on the segmented vessel, calculated velocity, and input aortic pressure. The standard operating procedure for AccuFFRangio computation is displayed in the Appendix 1.

\section{Statistical analysis}

In this investigation, the accuracy of AccuFFRangio was compared with the gold standard invasive FFR values using a cutoff value of $\leq 0.80$ for AccuFFRangio and a cutoff value of $\leq 0.80$ for standard wire-derived FFR. Data were analyzed on a per-vessel basis. Pearson correlation was used to quantify the correlation between AccuFFRangio and FFR. The diagnostic accuracy, sensitivity, specificity, positive predictive value (PPV), and negative predictive value (NPV) of AccuFFRangio were determined to assess the performance of the computed AccuFFRangio compared to measured FFR values. Bland-Altman analysis was used to analyze the agreement between computed AccuFFRangio and the measured FFR. The area under the curve (AUC) of receiver operating characteristic (ROC) analysis was used to assess the diagnostic accuracy of AccuFFRangio. The $\mathrm{V}$-plot of accuracies was also used as a sample-independent measure of the diagnostic performance of AccuFFRangio. All the statistical analyses were performed using MedCalc (MedCalc Software Inc., Belgium). 

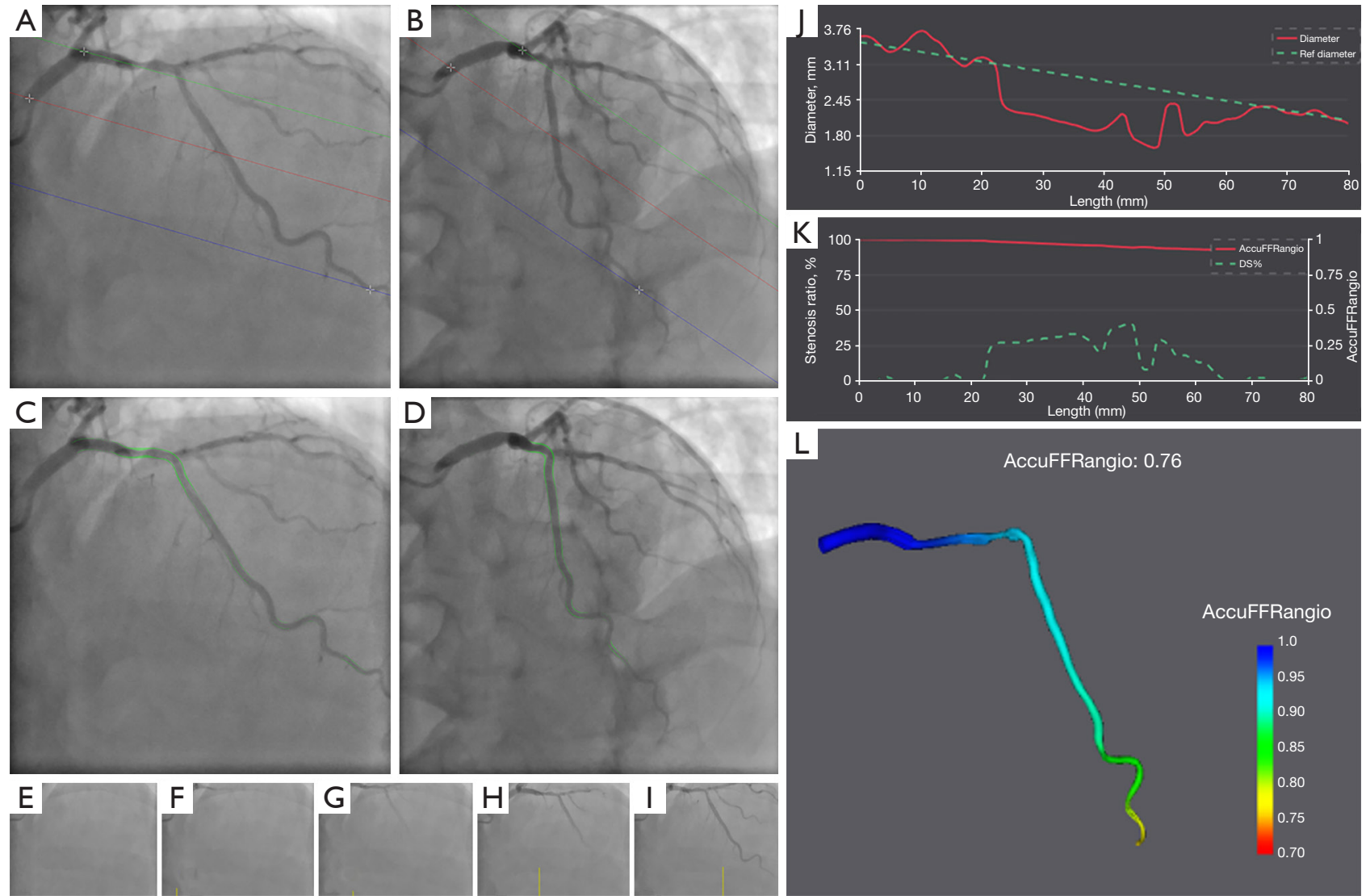

Figure 2 AccuFFRangio analysis of intermediate stenosis of a left anterior descending artery. (A-I) Consecutive angiographic image frames; $(\mathrm{J}, \mathrm{K})$ lumen diameter and stenosis ratio pullback; (L) AccuFFRangio was calculated as 0.76.

\section{Results}

\section{Patient characteristics}

In this study, a total of 320 patients with 320 interrogated vessels were enrolled. Table 1 summarizes the baseline clinical characteristics of the enrolled patients. The average age of the patients was $65 \pm 10$ years, and 99 (33.2\%) were women. Among the 298 interrogated vessels, AccuFFRangio was successfully assessed in all 298 vessels (100\%), FFR measurement was obtained in these 298 vessels (100\%). AccuFFRangio and FFR were both assessed in 298 vessels from 298 patients. The interrogated vessels had an average FFR of $0.83 \pm 0.10$. FFR $\leq 0.80$ was noted in 81 vessels (27.2\%). Vessel characteristics are presented in Table 2.

\section{Comparison of AccuFFRangio and invasively-measured FFR}

A good correlation was observed between AccuFFRangio and FFR, as shown in Figure 3, with a correlation coefficient of $\mathrm{r}=0.812(\mathrm{P}<0.001)$. There was good agreement between AccuFFRangio and FFR in the Bland-Altman plot, with a mean difference value of 0.001 (limits of agreement: -0.128 to 0.131 ) for per-vessel shown in Figure 4. The number of vessels with the absolute difference between AccuFFRangio and FFR falling outside of the $95 \% \mathrm{CI}$ (limits of agreement: -0.128 to 0.131 ) was $11(3.7 \%)$.

The accuracy of AccuFFRangio was $93.3 \%$ in this clinical investigation. The overall sensitivity, specificity, PPV, and NPV on a per-vessel basis were $92.6 \%, 93.5 \%, 84.3 \%$, and $97.1 \%$, respectively (Table 3). The AUC for AccuFFRangio was 0.96 (95\% CI: 0.932-0.98), as shown in Figure 5. The $\mathrm{V}$-plot of accuracies of AccuFFRangio (Figure 6) showed a very good diagnostic performance of AccuFFRangio across the spectrum of FFR values. Only a slight decrease in accuracy, which were still over $88 \%$, was observed between the FFR range of 0.75 and 0.85 , which was the so-called "gray zone". Moreover, the mean time for AccuFFRangio 
Table 1 Baseline patient characteristics $(\mathrm{n}=298)$

\begin{tabular}{|c|c|}
\hline Characteristics & Data \\
\hline Age, year & $65 \pm 10$ \\
\hline Male & $66.8 \%[199]$ \\
\hline Weight, kg & $68.4 \pm 34$ \\
\hline Height, cm & $165 \pm 9.9$ \\
\hline $\mathrm{BMI}, \mathrm{kg} / \mathrm{m}^{2}$ & $25.6 \pm 15.3$ \\
\hline Systolic blood pressure, $\mathrm{mmHg}$ & $132.3 \pm 17$ \\
\hline Diastolic blood pressure, $\mathrm{mmHg}$ & $76.1 \pm 11.6$ \\
\hline Heart rate, times/minute & $73 \pm 11$ \\
\hline Body temperature, ${ }^{\circ} \mathrm{C}$ & $36.9 \pm 0.2$ \\
\hline Breath, times/minute & $18.3 \pm 1.4$ \\
\hline Hypertension & $52.3 \%[156]$ \\
\hline Hypercholesterolemia & $10.7 \%[32]$ \\
\hline Kidney disease & $9.7 \%[29]$ \\
\hline Diabetes mellitus & $27.5 \%[82]$ \\
\hline Smoking history & $23.8 \%[71]$ \\
\hline Family history of coronary heart disease & $7 \%[21]$ \\
\hline Old myocardial infarction & $4.7 \%[14]$ \\
\hline Previous $\mathrm{PCl}$ & $21.1 \%[63]$ \\
\hline Previous CABG & $0.3 \%[1]$ \\
\hline
\end{tabular}

$\mathrm{BMI}$, body mass index; $\mathrm{PCl}$, percutaneous coronary intervention; CABG, coronary artery bypass graft.

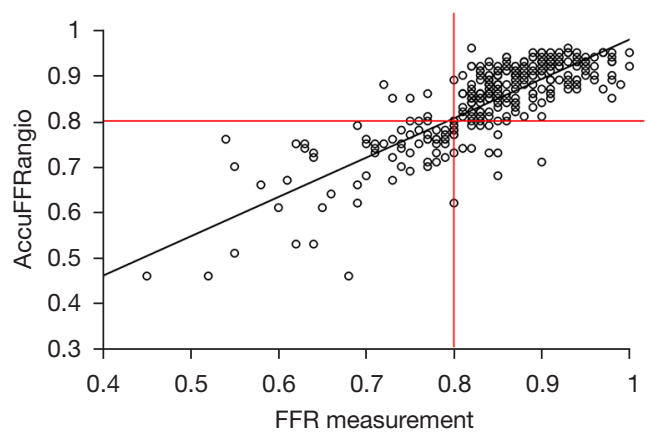

Figure 3 Correlation between FFR and AccuFFRangio. FFR, fractional flow reserve.
Table 2 Vessel characteristics $(\mathrm{n}=298)$

\begin{tabular}{lc}
\hline Characteristics & Data \\
\hline Vessels & \\
LAD & $62.8 \%[187]$ \\
LCX & $6.7 \%[20]$ \\
RCA & $29.2 \%[87]$ \\
$1^{\text {st }}$ OM & $1.3 \%[4]$ \\
Anatomy & \\
Diameter stenosis & $45.5 \% \pm 11.6 \%$ \\
$<50 \%$ & $50.7 \%[151]$ \\
$\geq 50 \%$ & $49.3 \%[147]$ \\
Physiology & \\
FFR (per vessel) & $0.83 \pm 0.10$ \\
Vessels with FFR $\leq 0.8$ & $27.2 \%[81]$ \\
Vessels with FFR $>0.8$ & $72.8 \%[217]$
\end{tabular}

$\mathrm{LAD}$, left anterior descending artery; LCX, left circumflex artery; $\mathrm{RCA}$, right coronary artery; OM, obtuse marginal artery; FFR, fractional flow reserve.

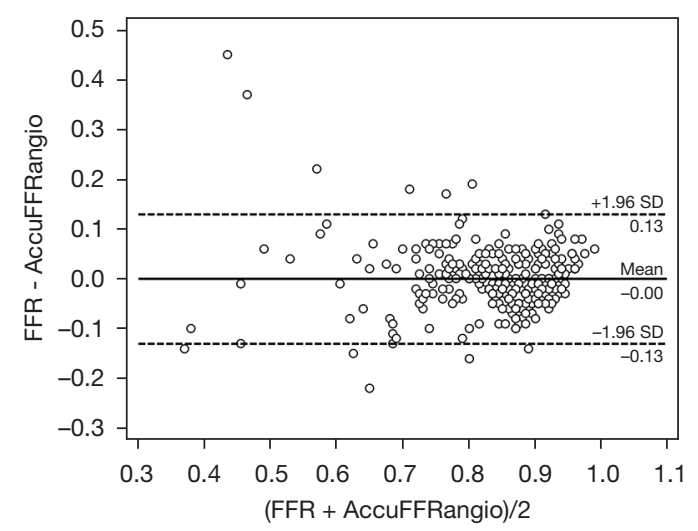

Figure 4 Bland-Altman plot of FFR and AccuFFRangio. FFR, fractional flow reserve. 
Table 3 Diagnostic performance of AccuFFRangio (per-vessel) $(\mathrm{n}=298)$

\begin{tabular}{lcc}
\hline & AccuFFRangio $\leq 0.8$ & $95 \% \mathrm{Cl}$ \\
\hline Accuracy, \% & 93.3 & $89.8-95.9$ \\
Sensitivity, \% & 92.6 & $84.6-97.2$ \\
Specificity, \% & 93.5 & $89.4-96.4$ \\
Positive likelihood ratio & 14.6 & $8.6-23.9$ \\
Negative likelihood ratio & 0.08 & $0.04-0.17$ \\
Disease prevalence, \% & 27.2 & $22.2-32.6$ \\
Positive predictive value, \% & 84.3 & $76.3-89.9$ \\
Negative predictive value, \% & 97.2 & $94.1-98.7$ \\
\hline
\end{tabular}

$\mathrm{Cl}$, confidence interval.

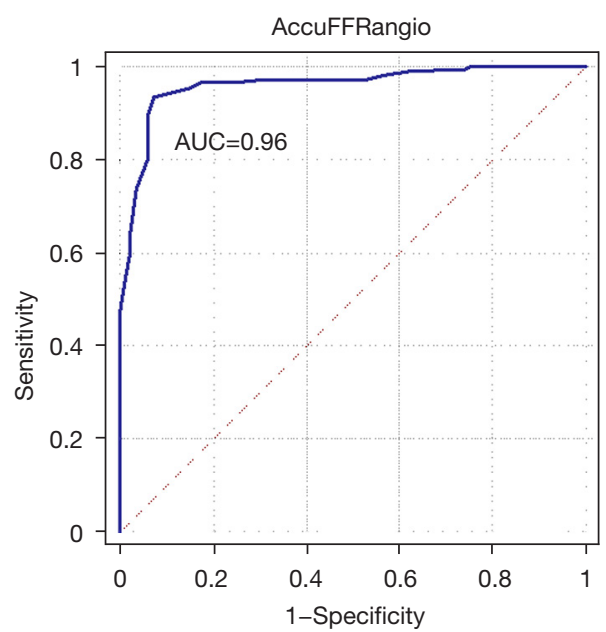

Figure 5 ROC curve of AccuFFRangio. ROC, receiver operating characteristic.

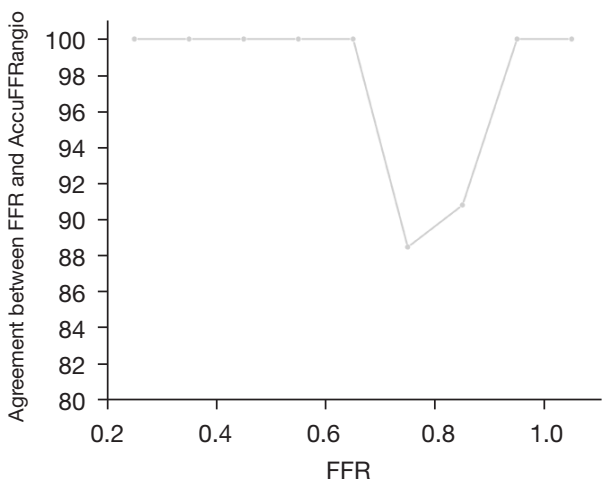

Figure 6 The V-plot of accuracies of AccuFFRangio. FFR, fractional flow reserve. assessment (including 3D angiographic reconstruction and frame count analysis) was $4.10 \pm 2.17 \mathrm{~min}$.

\section{Discussion}

This dual-center study observed that AccuFFRangio, an angiography-based approach for fast computation of FFR, demonstrated high feasibility and accuracy in identifying hemodynamically significant coronary stenosis. Compared with the anatomical assessment of coronary obstruction using ICA, AccuFFRangio substantially simplifies the diagnostic procedure of coronary angiography in identifying the coronary artery stenosis that leads to myocardial ischemia. This study also showed that AccuFFRangio computed using the contrast flow model, derived from coronary angiography without induction of hyperemia, had a high accuracy of $93.3 \%$ (95\% CI: 90.5-96.2\%) for the diagnosis of ischemia defined according to FFR $\leq 0.80$. In addition, the V-plot analysis demonstrated the good diagnostic performance of AccuFFRangio across the spectrum of disease severity, showing its potential for widespread application and reducing health care costs for numerous patients.

The wire-free FFR calculation based on ICA images is more accurate in evaluating the functional ischemia of coronary stenosis than the anatomical method. A 3D model of coronary stenosis of a single vessel was reconstructed through coronary angiographic images. Then, the TIMI frame count combined with 3D-QCA was used to calculate the average volume flow during hyperemia (20). A new FFR calculation method, angio-based FFR, was proposed using CFD or a numerical method to calculate pressure drop. The diagnostic performance of the angio-based FFR was evaluated with the FFR measured by the pressure wire as the reference standard. In 4 subsequent clinical trials $(10,16-18)$, the accuracies of quantitative flow ratio (QFR) were $85 \%, 83 \%, 86.8 \%$, and $92.4 \%$ in determining whether patients needed percutaneous coronary intervention (PCI) with a pressure wire measured with FFR $\leq 0.80$ as a reference. Several studies also used a similar method to reconstruct the $3 \mathrm{D}$ model of stenosis vessels, and the accuracy of angio-based FFR obtained by the numerical algorithm was about $85 \%(12,17)$. Similarly, a $3 \mathrm{D}$ model of multiple vessels was reconstructed from ICA images, with an overall accuracy of $92 \%(11,21)$.

In the present study, a new method improved 3D reconstruction and blood flow calculation, and the accuracy reached $93.3 \%$. From previous results, the accuracy of wire- 
free FFR calculated based on ICA images to determine whether PCI treatment is needed was from $80 \%$ to $95 \%$ $(10-12,17,18,21)$. For CTA-based FFR, the accuracy of noninvasive FFR was $80-90 \%(8,9)$. In comparison, the accuracy of QCA in determining whether patients need treatment was in the range of $50 \%$ to $70 \%$. Thus, the accuracy of FFR calculation based on ICA images is higher (10), mainly because the ICA images and the acquisition of blood flow velocity are more direct and real than those of CTA images. Early experiences with this technique by Morris et al. (22), Tu et al. (10), and Tröbs et al. (23), based on the geometric model reconstruction of the coronary artery and simulation of blood flow by solving the flow governing Navier-Stokes equations using the finite element method and CFD method, provided an accurate evaluation for calculating the pressure drop in coronary stenosis (23). The process may require 30 minutes of computation time and a dedicated computer to calculate a single vessel (no branches). However, this time is too long to meet the surgery requirements in the catheter lab, so a more rapid calculation method is needed.

One of the more accurate and rapid methods currently available is a mathematical algorithm known as the fast CFD method to calculate the pressure drop $\Delta \mathrm{P}$ at the coronary stenosis. $\Delta \mathrm{P}$ is calculated by multiplying the resistance by the flow volume $(\Delta \mathrm{P}=\mathrm{R} * \mathrm{Q})$. The resistance is determined by the minimum lumen area, reference vessel area, lesion length, and blood viscosity. There was no difference in the sensitivity and specificity of FFR obtained by CFD or mathematical methods. Besides, CFD simulations can be used to calibrate fast CFD methods. CFD was used in an initial study by Tu et al. (20), while a more effective mathematical approach was used in their subsequent study. The importance of applying this technique to the catheter lab is the need for quick calculations of FFR in about 5 min. In the FFRangio calculation $(11,24)$, the overall structure of the coronary vessels is reconstructed, and the vessel branches are simulated as circuit models. The blood flow is replaced by the current, and the blood pressure is equivalent to the voltage. The stenosis and diameter of the blood vessel affect the resistance of the circuit branch. Furthermore, the proximal and distal pressure ratio was analyzed as a coronary functional parameter, and FFRangio showed good diagnostic performance in clinical practice.

Several approaches were used to optimize the method adopted in the current study to improve the accuracy of the calculation. In the process of vessel remodeling correction, 3 polar lines are used, which can generate a more accurate $3 \mathrm{D}$ model of a target vessel. In the TIMI frame count method, the contrast medium frame number of coronary angiography images from 2 angles is averaged to avoid excessive deviation caused by the frame number of angiographic images from 1 angle. Most importantly, $\mathrm{Pa}$ (the mean pressure at the ostium of coronary arteries) is based on the average aortic pressure of the patient during the operation, which better reflects the patient-specific aortic pressure.

There are several limitations to this study. Not all the vessels were interrogated for the enrolled patients. The vessels with diameter stenosis $<30 \%$ or $>90 \%$ were not assessed because performing physiological assessments in such lesions was unnecessary. Side branches of bifurcation lesions defined as Medina type $1,1,1$ or $1,0,1$ were not evaluated. The generalizability of AccuFFRangio to the side branches of coronary bifurcation lesions requires further investigation. The data used in this study was at baseline, while hyperemic data can also be used for computation for this approach, though this may lead to adverse events caused by the administration of adenosine. The accuracy of AccuFFRangio was high in the present study; however, there were still numerical differences between AccuFFRangio and FFR. A prospective clinical trial comparing AccuFFRangio results with FFR measurement or randomized trials comparing clinical outcomes after using angio-based diagnostic procedures and standard diagnostic strategies has been planned. Furthermore, to compare the different methods of angio-based FFR, a larger head-to-head study needs to be conducted in the future.

\section{Conclusions}

This blinded, self-controlled, retrospective, and dualcenter clinical study aimed to evaluate the diagnostic accuracy of AccuFFRangio in the assessment of coronary stenosis. Compared with the gold standard invasive FFR, the accuracy, sensitivity, and specificity of AccuFFRangio in identifying hemodynamically significant coronary stenosis were $93.3 \%, 92.6 \%$, and $93.5 \%$, respectively. This clinical study demonstrates that AccuFFRangio is clinically feasible, and the performance is superior to angiographic assessment by $2 \mathrm{D}-\mathrm{QCA}$ for the evaluation of coronary artery stenosis.

\section{Acknowledgments}

Funding: This work was supported by the National Natural Science Foundation of China $(82170332,81320108003$, 
31371498, 81100141 , and 81570322), Zhejiang Provincial Public Welfare Technology Research Project (LGF20H020012), Zhejiang Provincial Key Research and Development Plan (2020C03016), Scientific Research Project of Zhejiang Education Department (Y201330290), and Major Medical and Health Science and Technology Plan of Zhejiang Province (WKJ-ZJ-1913).

\section{Footnote}

Reporting Checklist: The authors have completed the STARD reporting checklist. Available at https://dx.doi. org/10.21037/qims-21-463

Conflicts of Interest: All authors have completed the ICMJE uniform disclosure form (available at https://dx.doi. org/10.21037/qims-21-463). JJ receives research grants from National Natural Science Foundation of China (81570322) and Zhejiang Provincial Key Research and Development Plan (2020C03016). LT receives research grant from Zhejiang Provincial Key Research and Development Plan (2017C03034). CD receives research grant from Major Medical and Health Science and Technology Plan of Zhejiang Province (WKJ-ZJ-1913). XL receives research grant from National Natural Science Foundation of China (11802113). CL reeives research grants from Zhejiang Provincial Public Welfare Technology Research Project (LGF20H020012) and Scientific Research Project of Zhejiang Education Department (Y201330290). JX receives research grant from National Natural Science Foundation of China (81771242) and Stock/Stock Options of ArteryFlow Technology.

Ethical Statement: The authors are accountable for all aspects of the work in ensuring that questions related to the accuracy or integrity of any part of the work are appropriately investigated and resolved. The study was conducted in accordance with the Declaration of Helsinki (as revised in 2013). The study was approved by the Ethics Committees of The Second Affiliated Hospital, Zhejiang University School of Medicine and Zhejiang Hospital, and individual consent for this retrospective analysis was waived.

Open Access Statement: This is an Open Access article distributed in accordance with the Creative Commons Attribution-NonCommercial-NoDerivs 4.0 International License (CC BY-NC-ND 4.0), which permits the noncommercial replication and distribution of the article with the strict proviso that no changes or edits are made and the original work is properly cited (including links to both the formal publication through the relevant DOI and the license). See: https://creativecommons.org/licenses/by-nc-nd/4.0/.

\section{References}

1. Hoffmann U, Truong QA, Schoenfeld DA, Chou ET, Woodard PK, Nagurney JT, et al. Coronary CT angiography versus standard evaluation in acute chest pain. N Engl J Med 2012;367:299-308.

2. Meijboom WB, Van Mieghem CA, van Pelt N, Weustink A, Pugliese F, Mollet NR, Boersma E, Regar E, van Geuns RJ, de Jaegere PJ, Serruys PW, Krestin GP, de Feyter PJ. Comprehensive assessment of coronary artery stenoses: computed tomography coronary angiography versus conventional coronary angiography and correlation with fractional flow reserve in patients with stable angina. J Am Coll Cardiol 2008;52:636-43.

3. Pijls NH, De Bruyne B, Peels K, Van Der Voort PH, Bonnier HJ, Bartunek J Koolen JJ, Koolen JJ. Measurement of fractional flow reserve to assess the functional severity of coronary-artery stenoses. N Engl J Med 1996;334:1703-8.

4. Tonino PA, De Bruyne B, Pijls NH, Siebert U, Ikeno F, van' t Veer M, Klauss V, Manoharan G, Engstrøm T, Oldroyd KG, Ver Lee PN, MacCarthy PA, Fearon WF; FAME Study Investigators. Fractional flow reserve versus angiography for guiding percutaneous coronary intervention. N Engl J Med 2009;360:213-24.

5. De Bruyne B, Pijls NH, Kalesan B, Barbato E, Tonino PA, Piroth Z, et al. Fractional flow reserve-guided PCI versus medical therapy in stable coronary disease. N Engl J Med 2012;367:991-1001.

6. Fihn SD, Gardin JM, Abrams J, Berra K, Blankenship JC, Dallas AP, et al. 2012 ACCF/AHA/ACP/AATS/PCNA/ SCAI/STS Guideline for the diagnosis and management of patients with stable ischemic heart disease: a report of the American College of Cardiology Foundation/American Heart Association Task Force on Practice Guidelines, and the American College of Physicians, American Association for Thoracic Surgery, Preventive Cardiovascular Nurses Association, Society for Cardiovascular Angiography and Interventions, and Society of Thoracic Surgeons. J Am Coll Cardiol 2012;60:e44-e164.

7. Koo BK, Erglis A, Doh JH, Daniels DV, Jegere S, Kim HS, Dunning A, DeFrance T, Lansky A, Leipsic J, Min JK. Diagnosis of ischemia-causing coronary stenoses 
by noninvasive fractional flow reserve computed from coronary computed tomographic angiograms. Results from the prospective multicenter DISCOVER-FLOW (Diagnosis of Ischemia-Causing Stenoses Obtained Via Noninvasive Fractional Flow Reserve) study. J Am Coll Cardiol 2011;58:1989-97.

8. Nakazato R, Park HB, Berman DS, Gransar H, Koo BK, Erglis A, Lin FY, Dunning AM, Budoff MJ, Malpeso J, Leipsic J, Min JK. Noninvasive fractional flow reserve derived from computed tomography angiography for coronary lesions of intermediate stenosis severity: results from the DeFACTO study. Circ Cardiovasc Imaging 2013;6:881-9.

9. Nørgaard BL, Leipsic J, Gaur S, Seneviratne S, Ko BS, Ito $\mathrm{H}$, et al. Diagnostic performance of noninvasive fractional flow reserve derived from coronary computed tomography angiography in suspected coronary artery disease: the NXT trial (Analysis of Coronary Blood Flow Using CT Angiography: Next Steps). J Am Coll Cardiol 2014;63:1145-55.

10. Tu S, Westra J, Yang J, von Birgelen C, Ferrara A, Pellicano M, Nef H, Tebaldi M, Murasato Y, Lansky A, Barbato E, van der Heijden LC, Reiber JHC, Holm NR, Wijns W; FAVOR Pilot Trial Study Group. Diagnostic Accuracy of Fast Computational Approaches to Derive Fractional Flow Reserve From Diagnostic Coronary Angiography: The International Multicenter FAVOR Pilot Study. JACC Cardiovasc Interv 2016;9:2024-35.

11. Pellicano M, Lavi I, De Bruyne B, Vaknin-Assa H, Assali A, Valtzer O, Lotringer Y, Weisz G, Almagor Y, Xaplanteris P, Kirtane AJ, Codner P, Leon MB, Kornowski R. Validation Study of Image-Based Fractional Flow Reserve During Coronary Angiography. Circ Cardiovasc Interv 2017;10:e005259.

12. Westra J, Andersen BK, Campo G, Matsuo H, Koltowski L, Eftekhari A, et al. Diagnostic Performance of InProcedure Angiography-Derived Quantitative Flow Reserve Compared to Pressure-Derived Fractional Flow Reserve: The FAVOR II Europe-Japan Study. J Am Heart Assoc 2018;7:009603.

13. Zuo W, Zhang R, Yang M, Ji Z, He Y, Su Y, Qu Y, Tao Z, Ma G. Clinical prediction models of fractional flow reserve: an exploration of the current evidence and appraisal of model performance. Quant Imaging Med Surg 2021;11:2642-57.

14. Fairbairn TA, Nieman K, Akasaka T, Nørgaard BL, Berman DS, Raff G, et al. Real-world clinical utility and impact on clinical decision-making of coronary computed tomography angiography-derived fractional flow reserve: lessons from the ADVANCE Registry. Eur Heart J 2018;39:3701-11.

15. Patel MR, Nørgaard BL, Fairbairn TA, Nieman K, Akasaka T, Berman DS, et al. 1-Year Impact on Medical Practice and Clinical Outcomes of FFRCT: The ADVANCE Registry. JACC Cardiovasc Imaging 2020;13:97-105.

16. Asano T, Katagiri Y, Collet C, Tenekecioglu E, Miyazaki Y, Sotomi Y, et al. Functional comparison between the BuMA Supreme biodegradable polymer sirolimus-eluting stent and a durable polymer zotarolimus-eluting coronary stent using quantitative flow ratio: PIONEER QFR substudy. EuroIntervention 2018;14:e570-9.

17. Westra J, Tu S, Winther S, Nissen L, Vestergaard MB, Andersen BK, et al. Evaluation of Coronary Artery Stenosis by Quantitative Flow Ratio During Invasive Coronary Angiography: The WIFI II Study (WireFree Functional Imaging II). Circ Cardiovasc Imaging 2018;11:e007107.

18. Xu B, Tu S, Qiao S, Qu X, Chen Y, Yang J, Guo L, Sun Z, Li Z, Tian F, Fang W, Chen J, Li W, Guan C, Holm NR, Wijns W, Hu S. Diagnostic Accuracy of AngiographyBased Quantitative Flow Ratio Measurements for Online Assessment of Coronary Stenosis. J Am Coll Cardiol 2017;70:3077-87.

19. Jiang J, Feng L, Li C, Xia Y, He J, Leng X, Dong L, Hu $\mathrm{X}$, Wang J, Xiang J. Fractional flow reserve for coronary stenosis assessment derived from fusion of intravascular ultrasound and X-ray angiography. Quant Imaging Med Surg 2021;11:4543-55.

20. Tu S, Barbato E, Köszegi Z, Yang J, Sun Z, Holm NR, Tar B, Li Y, Rusinaru D, Wijns W, Reiber JH. Fractional flow reserve calculation from 3-dimensional quantitative coronary angiography and TIMI frame count: a fast computer model to quantify the functional significance of moderately obstructed coronary arteries. JACC Cardiovasc Interv 2014;7:768-77.

21. Fearon WF, Achenbach S, Engstrom T, Assali A, Shlofmitz R, Jeremias A, Fournier S, Kirtane AJ, Kornowski R, Greenberg G, Jubeh R, Kolansky DM, McAndrew T, Dressler O, Maehara A, Matsumura M, Leon MB, De Bruyne B; FAST-FFR Study Investigators. Accuracy of Fractional Flow Reserve Derived From Coronary Angiography. Circulation 2019;139:477-84.

22. Morris PD, Ryan D, Morton AC, Lycett R, Lawford PV, Hose DR, Gunn JP. Virtual fractional flow reserve from coronary angiography: modeling the significance of 
coronary lesions: results from the VIRTU-1 (VIRTUal Fractional Flow Reserve From Coronary Angiography) study. JACC Cardiovasc Interv 2013;6:149-57.

23. Tröbs M, Achenbach S, Röther J, Redel T, Scheuering M, Winneberger D, Klingenbeck K, Itu L, Passerini T, Kamen A, Sharma P, Comaniciu D, Schlundt C. Comparison of Fractional Flow Reserve Based on Computational Fluid
Dynamics Modeling Using Coronary Angiographic Vessel Morphology Versus Invasively Measured Fractional Flow Reserve. Am J Cardiol 2016;117:29-35.

24. Kornowski R, Lavi I, Pellicano M, Xaplanteris P, VakninAssa H, Assali A, Valtzer O, Lotringer Y, De Bruyne B. Fractional Flow Reserve Derived From Routine Coronary Angiograms. J Am Coll Cardiol 2016;68:2235-7.
Cite this article as: Jiang J, Tang L, Du C, Leng X, He J, Hu Y, Dong L, Sun Y, Li C, Xiang J, Wang J. Diagnostic performance of AccuFFRangio in the functional assessment of coronary stenosis compared with pressure wire-derived fractional flow reserve. Quant Imaging Med Surg 2022;12(2):949-958. doi: 10.21037/qims-21-463 


\section{Appendix 1 Standard operating procedure for AccuFFRangio computation}

\section{Loading Images}

AccuFFRangio supports image files with compressed or uncompressed DICOM format.

Proper angiographic projections are required:

(I) Image acquisition speed at least 15 frames per second;

(II) The 2 angiographic images at least $25^{\circ}$ apart;

(III) Target vessel without severe overlap/foreshortening in the selected images;

(IV) Projection is in optimal perspective viewing angle;

(V) Sufficient injected contrast for image analysis;

(VI) Sufficient injected contrast for frame count evaluation.

\section{Viewports}

The Video Viewports can display 2 matching images of the same patient. The projection angle of these 2 images must be at least $25^{\circ}$ and the frame rate should be at least 15 frames per second.

\section{ECG Diagram}

If available, the ECG diagrams of images will be shown at the bottom of the viewports (Figure S1).

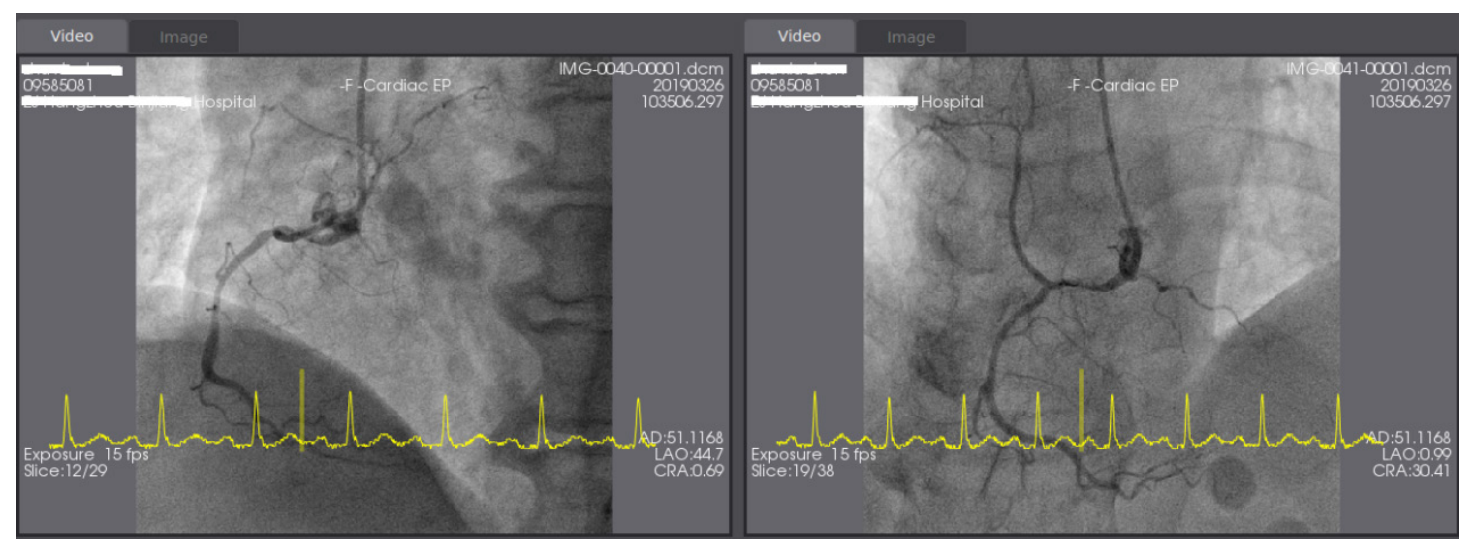

Figure S1 ECG diagrams of images of the same patient.

\section{AccuFFRangio Analysis}

\section{Frame Selection}

The appropriate frames for analysis should be determined (Figure S2):

(I) The target vessel without severe overlap;

(II) The entire vessel segment is injected with sufficient contrast;

(III) Frames are selected at the end-diastolic phase; 


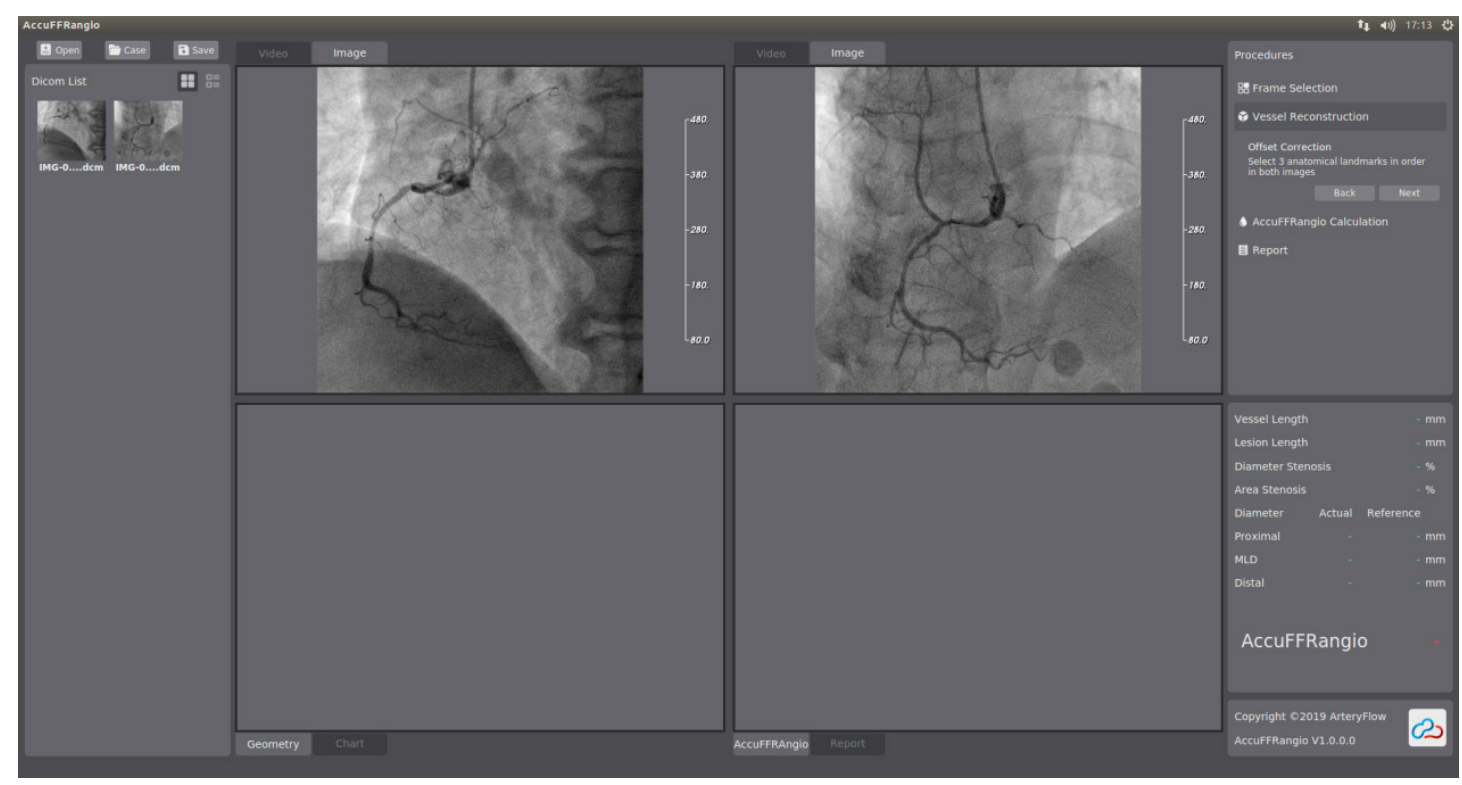

Figure S2 Frame selection of the entire vessel segment at the end-diastolic phase with sufficient contrast.

\section{Vessel Reconstruction}

\section{Offset Correction}

The first step of AccuFFRangio calculation is isocenter offset correction, which aims to correct the angiographic system distortions. Then, the corresponding relationship is created for these 2 images. In other words, for any point in the left viewport, we can find the corresponding point in the right viewport.

To apply offset correction:

(I) In the upper left-hand viewport, left-click to place 3 reference mark points.

(II) In the upper right-hand viewport, left-click to place reference mark points at the corresponding location.

Besides, make sure to:

(I) Identify the mark point that is easily identified in both of the 2 projections (i.e., a bifurcation, localized stenosis, or the offspring of a side branch);

(II) If using a side branch:

a) Select a side branch that departs perpendicularly from the main branch if possible;

b) Place the reference mark points on the main vessel;

If the generated epipolar lines go through the reference mark points in both the left-hand and right-hand viewports, offset correction is successful (Figure S3). Otherwise, it fails to correct the offset. Then, users should revise the position of the chosen reference mark points or select different locations for the reference mark points.

\section{Target Vessel Indication}

This step aims to define the pathline and contours of the vessel of interest in the upper left-hand and upper right-hand viewports.

You should indicate the proximal and distal points for the target vessel to generate the pathline:

(I) Users should left-click on the upper left-hand viewport to define the proximal and distal points of the vessel of interest.

(II) Users should left-click on the upper right-hand viewport to define the proximal point and distal point of the vessel of interest.

(III) If the pathline is not located in the region of the vessel, the user can left-click and drag the pathline to be correctly 


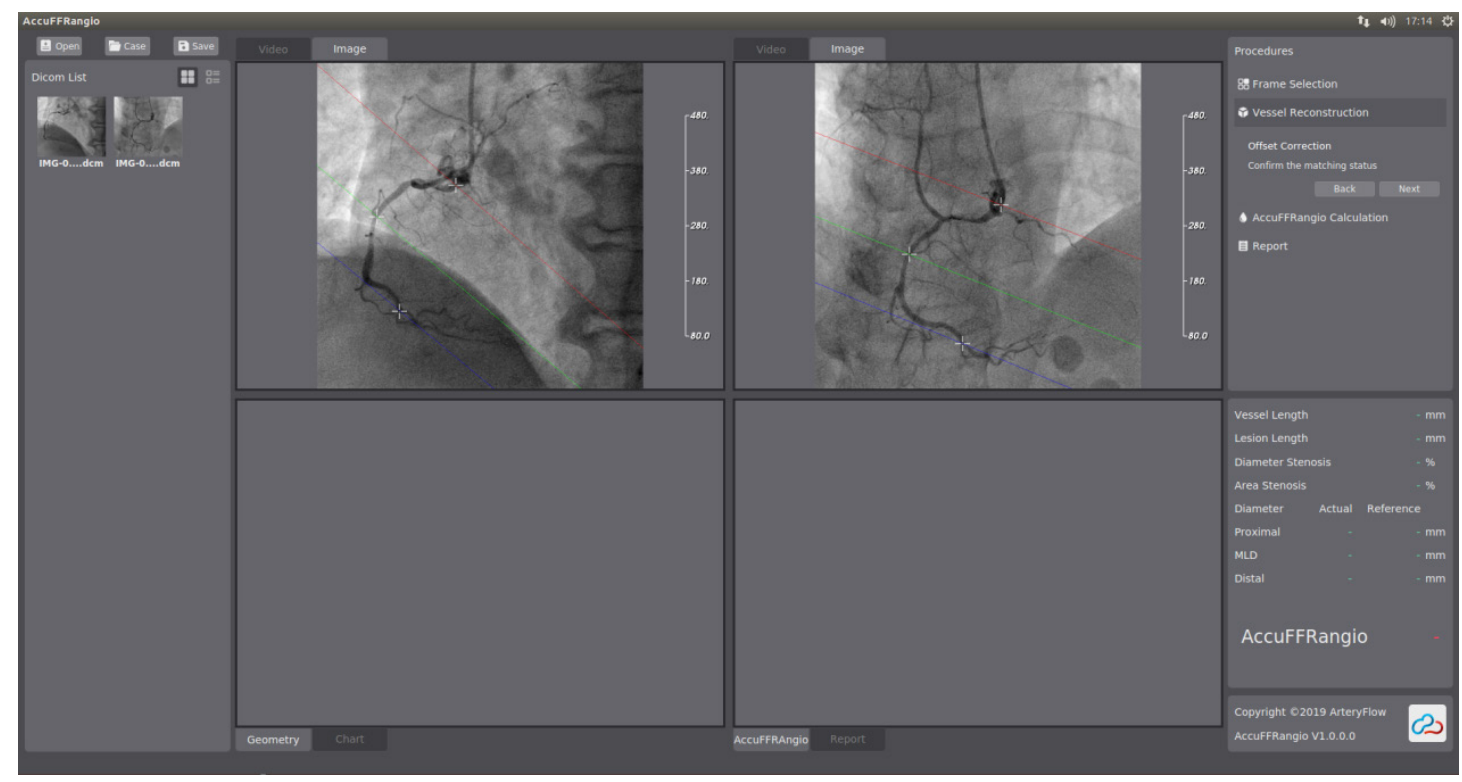

Figure S3 Offset correction.

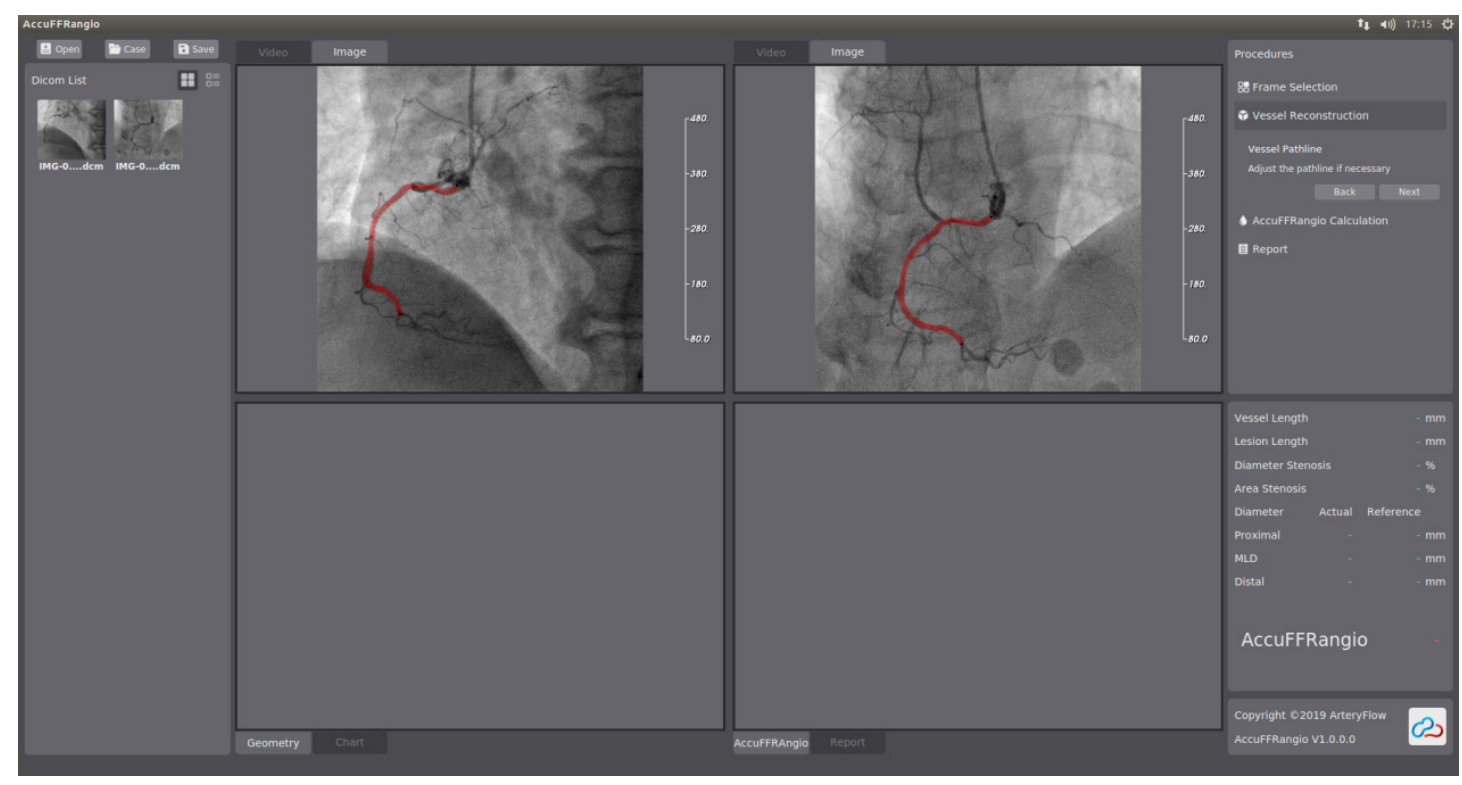

Figure S4 The pathline of the target vessel.

placed.

If successful, the pathline is shown correctly in the left and right Viewports (in Figure S4).

\section{Lumen Contouring}

To generate contours:

(I) Click in the Procedures toolbar (Figure S5). The contours of the target vessel needing to be segmented will be generated automatically and shown in the upper left-hand and upper right-hand viewport. 


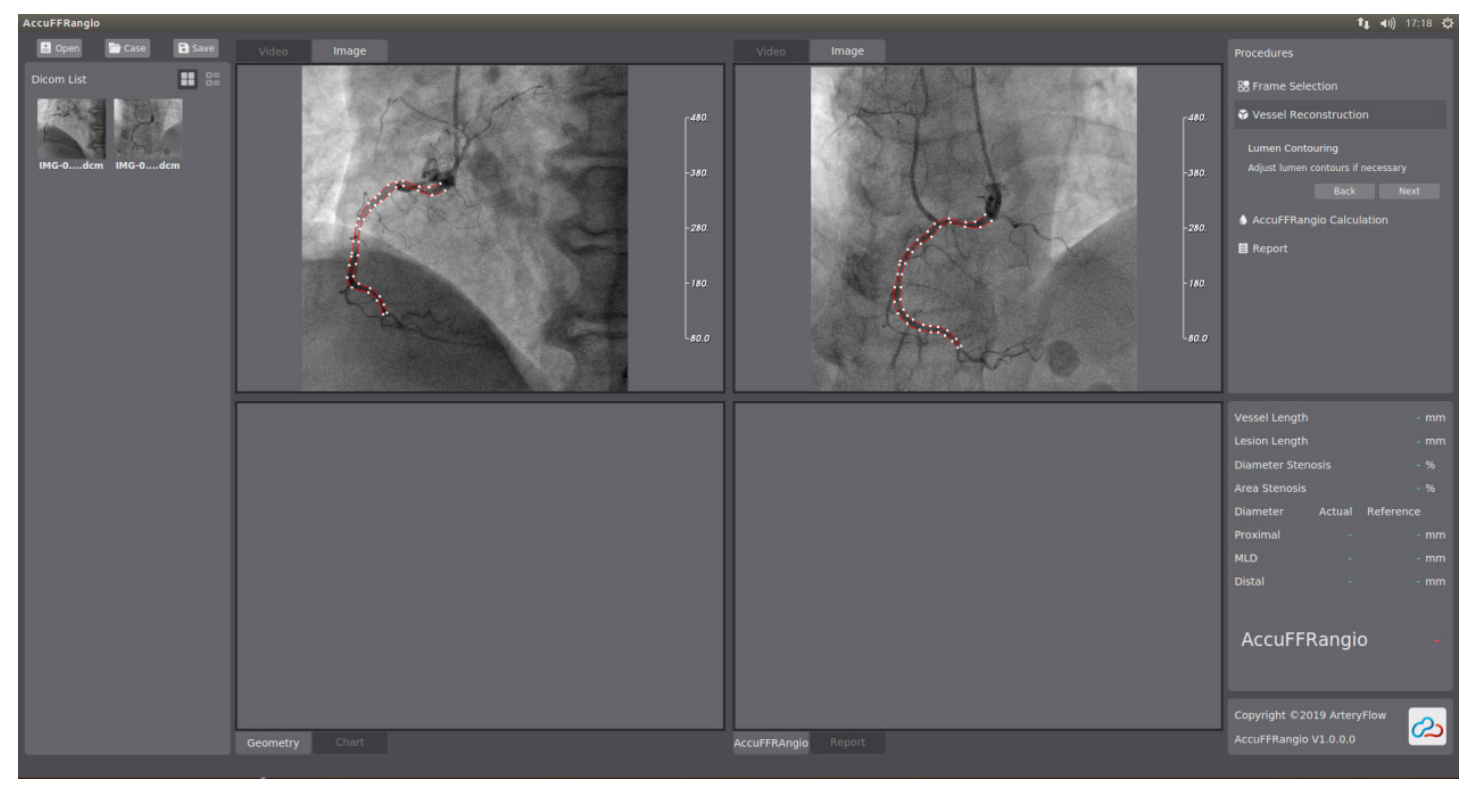

Figure S5 Contours of the vessel.

(II) If needed, the user can left-click and drag the boundary points on the contours to fit the boundary of the vessel.

Users can add boundary points to define the boundary of the target vessel by left-clicking on the contours. Users can also delete points on the contours by pressing the 'Delete' key.

When fitting the red contour lines to the border of the lumen, pay special attention to:

(I) Ensure non-existing narrowing in the proximal and distal ends

(II) Correct contouring of the target vessels

(III) Side branches and overlap

(IV) Ensure that contours are correct in all segments and also non-target segments as it influences AccuFFRangio calculation

Figure $S 5$ shows the final contours of the vessel in the upper left-hand and upper right-hand viewports.

\section{Forced Correspondence}

In this step, the lesion of the vessel segment will correspond forcedly and be displayed as a green region. Clicking in the Procedures pane (Figure S6), the lesion will be generated and correspond automatically, and will be shown in the upper lefthand and right-hand viewports.

Then, click in the Procedures pane, and the 3D geometry of the vessel will be generated and displayed in the Geometry Viewport (Figure S7).

\section{Reference Vessel}

The reference vessel is generated based on the three-dimensional reconstruction of the real vessel segment automatically. Any part of the contoured lumen narrowing than the reference vessel is marked the green region as plaque. The reference function can be seen in the lower left-hand pane, in which a red chart means vessel diameter distribution along the pathline of the vessel and the green dashed line represents the reference function (Figure S11).

The 3D reference vessel will be displayed in the Geometry Viewport. Besides, QCA results will be calculated and shown in the Properties pane. 


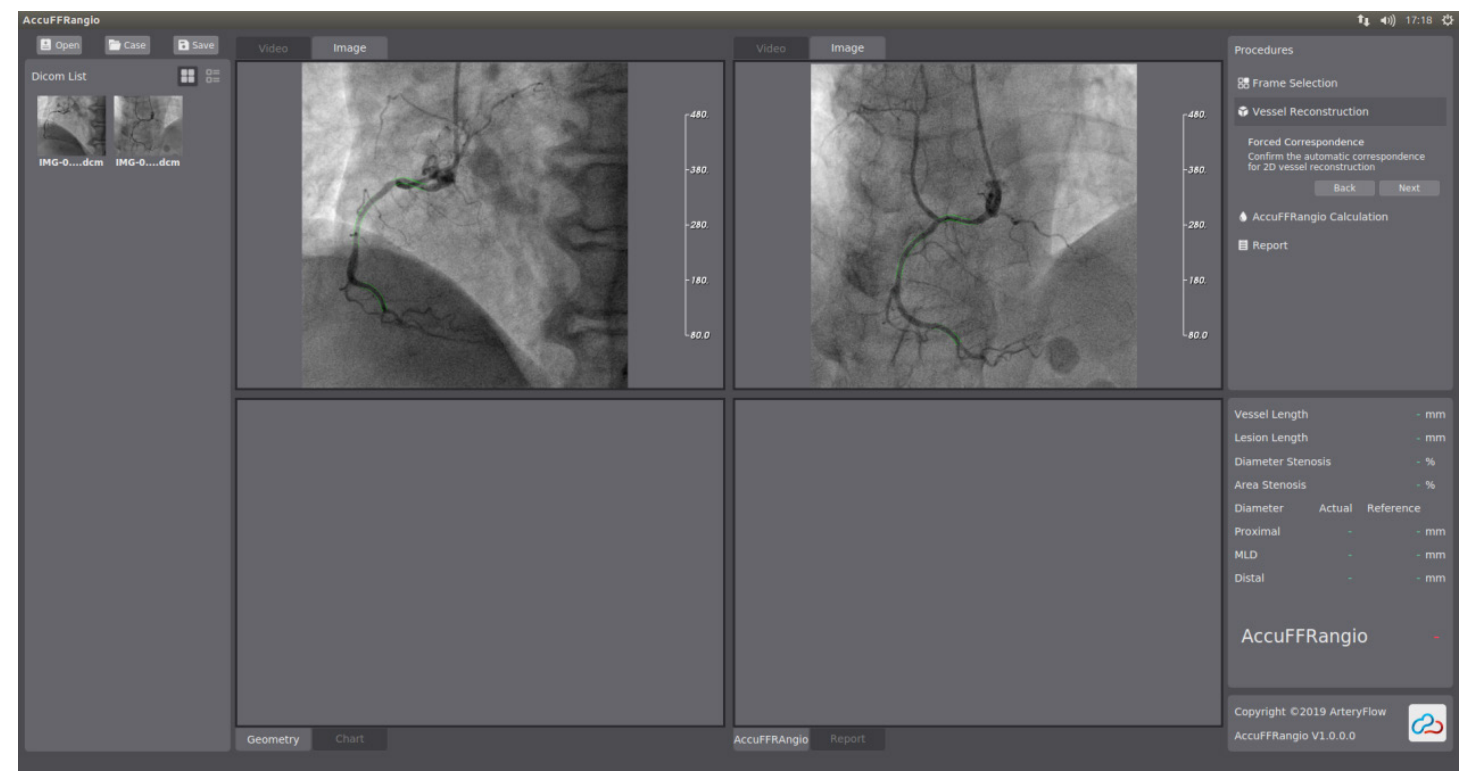

Figure S6 Stenosis of the vessel.

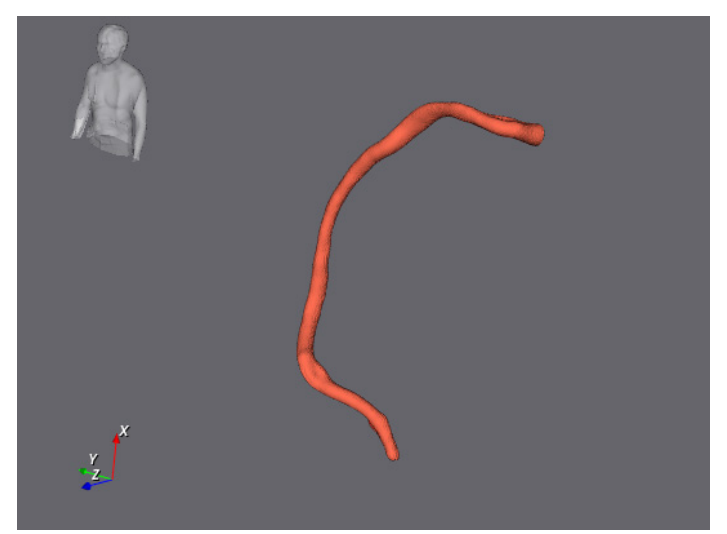

Figure S7 Geometry Viewport.
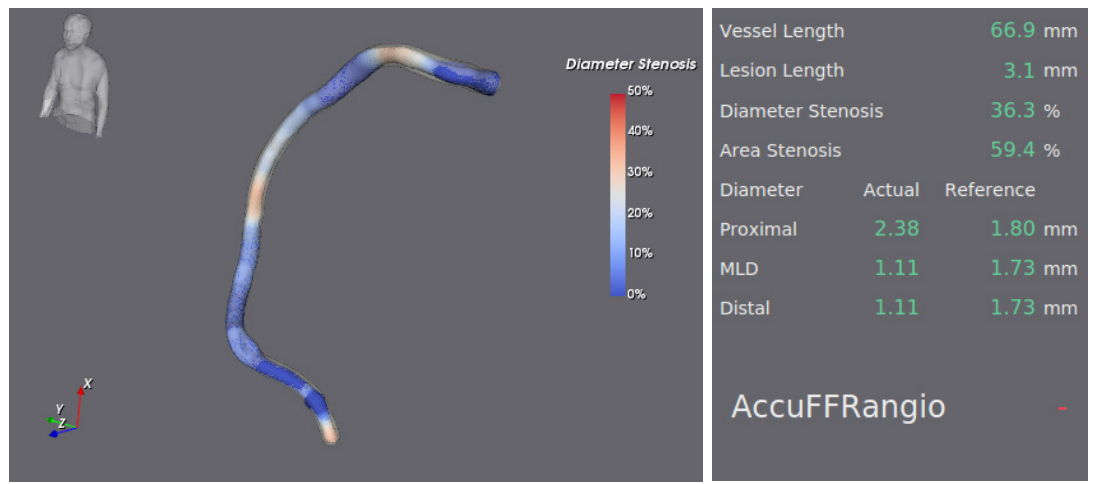

Figure S8 Geometry Viewport and Properties pane. 


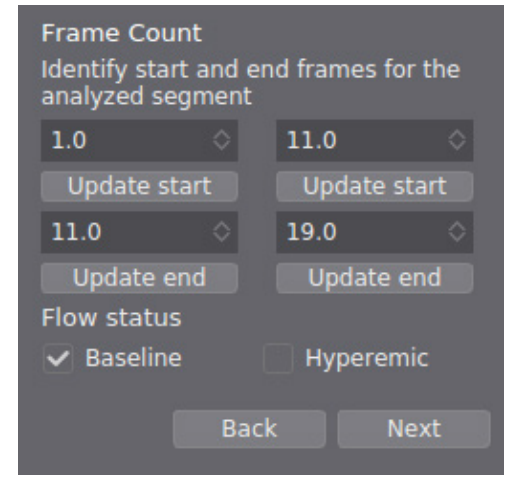

Figure S9 Aortic Pressure input.

\section{AccuFFRangio Calculation}

This step details the calculation of AccuFFRangio.

\section{Frame Count}

To derive the flow velocity throughout the segmented vessel and to obtain a more accurate AccuFFRangio value, the frame counting method is applied (Figure S9).

To perform the frame count:

(I) Rotate the mouse scroll wheel to navigate through the frames until the start frames in both the upper left-hand and upper right-hand viewports are found. Press the button to record the index of the start frame. The start frame is defined as the frame in which the contrast arrives at the proximal pathline point.

(II) Rotate the mouse scroll wheel to navigate further through the frames until the end frames in the upper left-hand and upper right-hand video views are found. Press the button to record the index of the end frame. The end frame is defined as the frame in which the contrast arrives at the distal pathline point. Users can also enter the index of the start frame and end frame manually in the input box, as seen in the above figure.

According to whether the patient was injected with vasodilators, you should select the radio button to specify the patient state: Hyperemia or Baseline.

\section{Aortic Pressure}

You should specify the aortic pressure to calculate the AccuFFRangio value obtained from the catheter lab's monitoring panel (Figure S10).

\section{AccuFFRangio Results}

The last step is to determine AccuFFRangio values based on the above operations.

To determine the AccuFFRangio results (Figure S11):

(I) Click on the button, the AccuFFRangio value at the distal end of the segmented vessel is shown at the lower right corner of the Properties pane.

The vessel geometry with different colors representing different AccuFFRangio values is demonstrated in the lower right-hand viewport.

(II) Users can choose a position at the vessel geometry in the lower right-hand viewport by left-clicking and obtain the AccuFFRangio value.

After the AccuFFRangio calculation, you can edit detailed information in the report.

Then, click on the Report module to generate a report. 2D stenosis of the vessel in 2 projections, the vessel with 


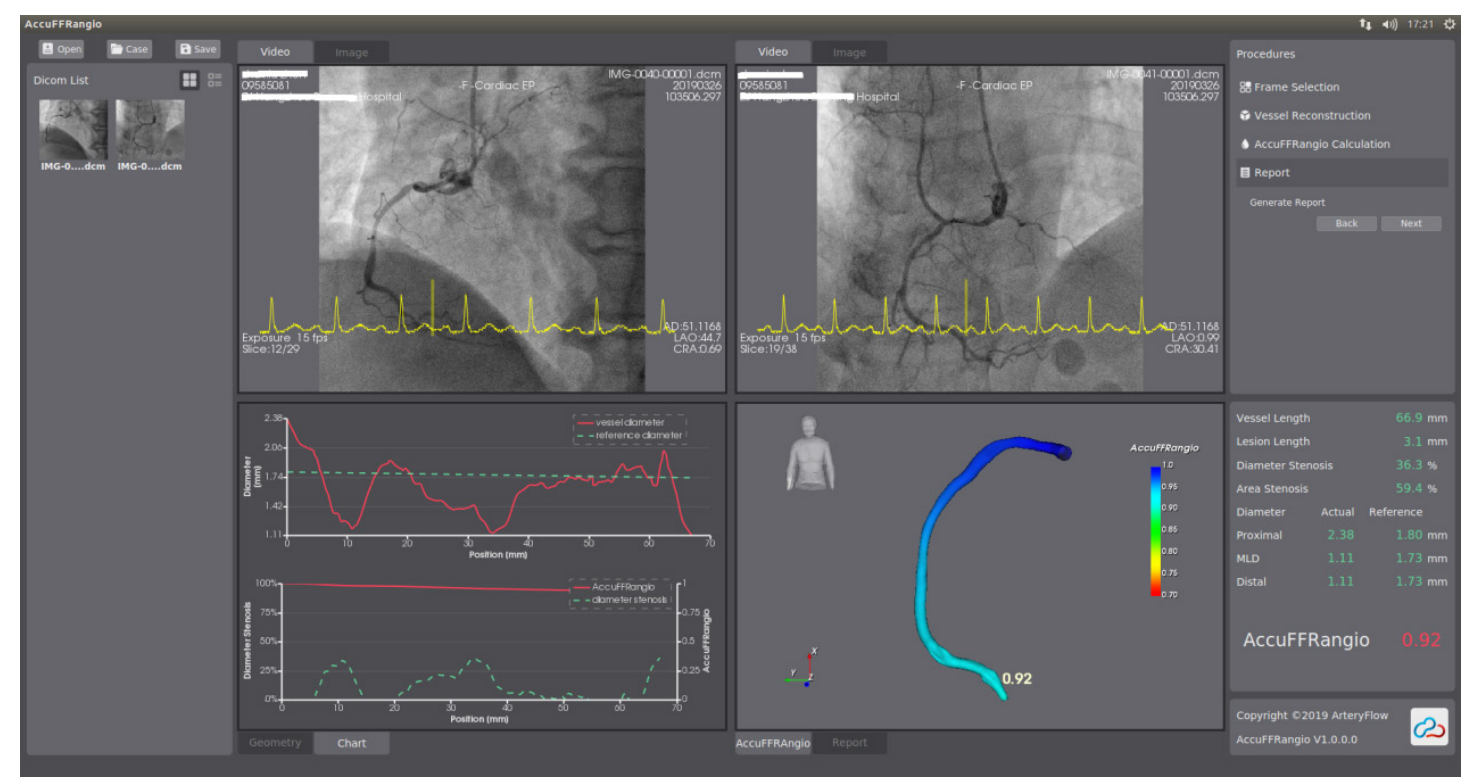

Figure S11 AccuFFRangio results.

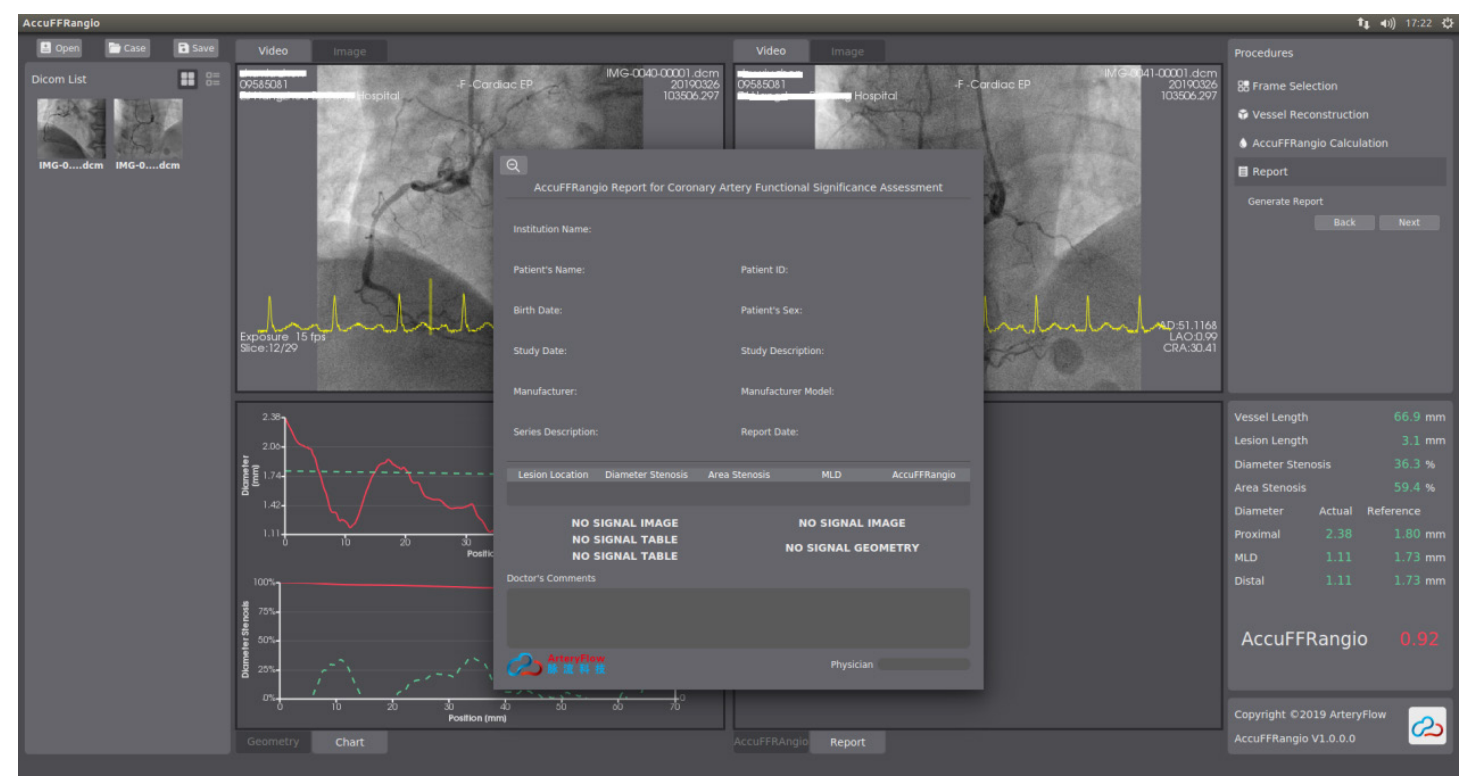

Figure S12 Edit the report.

AccuFFRangio contour distribution, 2 charts representing vessel diameter vs. position and reference vessel diameter $v s$. position, and AccuFFRangio value $v s$. position and diameter stenosis $v s$. position will be presented in the report. The report can also be exported to PDF files. Figure S12 is the final report with detailed information. 